\title{
EFFECT OF TEMPERATURE AND PRESSURE ON SEGMENTAL RELAXATION IN POLYMETHYLPHENYLSILOXANE
}

\author{
S. Pawlus, S. J Rzoska, J. Ziolo, M. Paluch \\ Institute of Physics, University of Silesia, Uniwersytecka 4, 40-007 Katowice, Poland \\ C.M. ROLAND* \\ Naval Research Laboratory, Chemistry Division, Code 6120, Washington, DC 20375-5342
}

\begin{abstract}
Segmental relaxation in a series of polymethylphenylsiloxanes (PMPS) was studied using dielectric spectroscopy. The measurements covered a temperature range of more than $40 \mathrm{deg}$ at pressures from ambient to $115 \mathrm{MPa}$. The results confirmed that the shape of the loss peak is independent of temperature, pressure and molecular weight. Consequently, the $\mathrm{T}_{\mathrm{g}}$-scaled dependence of the relaxation times was also independent of molecular weight. The pressure dependence of the relaxation times was characterized by means of the activation volume. This quantity changes markedly with pressure at a given temperature. However, the activation volume at the respective glass transition temperatures of the PMPS are essentially invariant to molecular weight. Finally, we measured the dependence of $T_{g}$ on pressure, with the results well-described by the Andersson equation.
\end{abstract}

\section{INTRODUCTION}

The transition of a rubber or amorphous liquid into a glass can be brought about by a variety of means, including cooling and increased hydrostatic pressure. The dynamics in the supercooled regime prior to the structural arrest associated the glassy state are complex and not fully understood. Determining the connection between these motions (referred to as local segmental relaxation in polymers) and the chemical and thermodynamic properties is essential to establishing a molecular basis for the physical properties of a material. Experimental efforts along these lines usually focus on the effect of temperature, with correlations having been established between the temperature dependence of the relaxation times and both the shape of the relaxation function $^{1-3}$ and the chemical structure. ${ }^{2,4-6}$

In developing structure/property relationships, pressure dependences can be equally informative, and recently there has been a burgeoning use of pressure as an experimental variable..$^{7-13}$ Relaxation measurements under high pressure allow the relative contributions of volume and thermal energy to be quantified, ${ }^{14}$ which is essential information for any theory of the glass transition.

Siloxane polymers are commercially very important, finding applications ranging from lubricants and heat exchange fluids to medical devices and cosmetic products. Siloxane materials are also intriguing from a scientific viewpoint. Their low glass transition temperature in combination with excellent thermal stability enables measurements over an enormous range of temperatures. ${ }^{15-17}$ Monodisperse polysiloxanes are readily available in a range of molecular weights, and with a variety of chemical side-groups. In this paper, we describe dielectric measurements on polymethylphenylsiloxanes (PMPS) of varying molecular weight, carried out as a function of both temperature and pressure. In earlier work, ${ }^{18,19}$ we had compared the segmental relaxation properties of PMPS and polymethyltolylsiloxane (PMTS). At ambient pressure, their respective time dependences (i.e., shape of the relaxation function) and $\mathrm{T}_{\mathrm{g}}$-normalized temperature dependences are equivalent, consistent with their very similar chemical structures. However, the pressure dependences of the dielectric relaxation differed markedly..$^{19}$ Herein, we report results for PMPS over a molecular weight range of from 12.6 to $121 \mathrm{~kg} / \mathrm{mol}$. The measurements were car-

* Corresponding author: Ph: 202-767-1769; Fax: 202-767-0594; email: roland@nrl.navy.mil 


\section{EFFECT OF TEMPERATURE AND PRESSURE ON SEGMENTAL RELAXATION 1107}

ried out over a temperature range of from $\mathrm{T}_{\mathrm{g}}$ to $c a$. 40 degrees above $\mathrm{T}_{\mathrm{g}}$, at pressures from ambient to $115 \mathrm{MPa}$. The effect of pressure on the relaxation times and the relaxation function, as well as any dependence on chain length, is analyzed.

\section{EXPERIMENTAL}

The polymers, from various sources, are listed in Table I. The dielectric measurements were carried out using a Novo-Control $\mathrm{GmbH}$ dielectric spectrometer. The dielectric permittivity, $\varepsilon^{*}(\omega)=\varepsilon^{\prime}(\omega)-i \varepsilon^{\prime \prime}(\omega)$, was measured in the frequency range from 0.01 to $3 \times 10^{6} \mathrm{~Hz}$. For ambient pressure measurements, the sample was contained between parallel plates (diameter $10 \mathrm{~mm}$, gap $0.1 \mathrm{~mm}$ ), with temperature controlled to better than $0.1 \mathrm{~K}$ using a nitrogen-gas cryostat.

For the high-pressure measurements, the parallel plate capacitor, after filling, was sealed and mounted inside a Teflon ring spacer. This assembly was then placed in the high-pressure cell. Pressure was exerted via silicone fluid, using a chamber with a piston in contact with a hydraulic press. Pressure was measured by a Nova Swiss tensometric pressure meter (resolution $=0.1$ $\mathrm{MPa}$ ). The temperature was controlled to $0.1 \mathrm{~K}$ by means of liquid flowing from a thermostatic bath.

\section{RESULTS}

\section{ATMOSPHERIC PRESSURE RESULTS}

Displayed in Figure 1 are representative dielectric constant and loss spectra obtained at ambient pressure for PMPS $\left(\mathrm{M}_{\mathrm{w}}=52.8 \mathrm{~kg} / \mathrm{mol}\right)$. With decreasing temperature, there is a marked shifting of the spectra to lower frequency, along with a systematic increase in the dielectric strength, $\Delta \varepsilon$. This behavior is expected for an amorphous polymer near $\mathrm{T}_{\mathrm{g}}$, and very similar to that of the other samples, having different molecular weights. In fact, as shown in Figure 2, when compared at temperatures for which their respective $\tau$ are equal, the shape of the dielectric relaxation function for PMPS is independent of molecular weight.

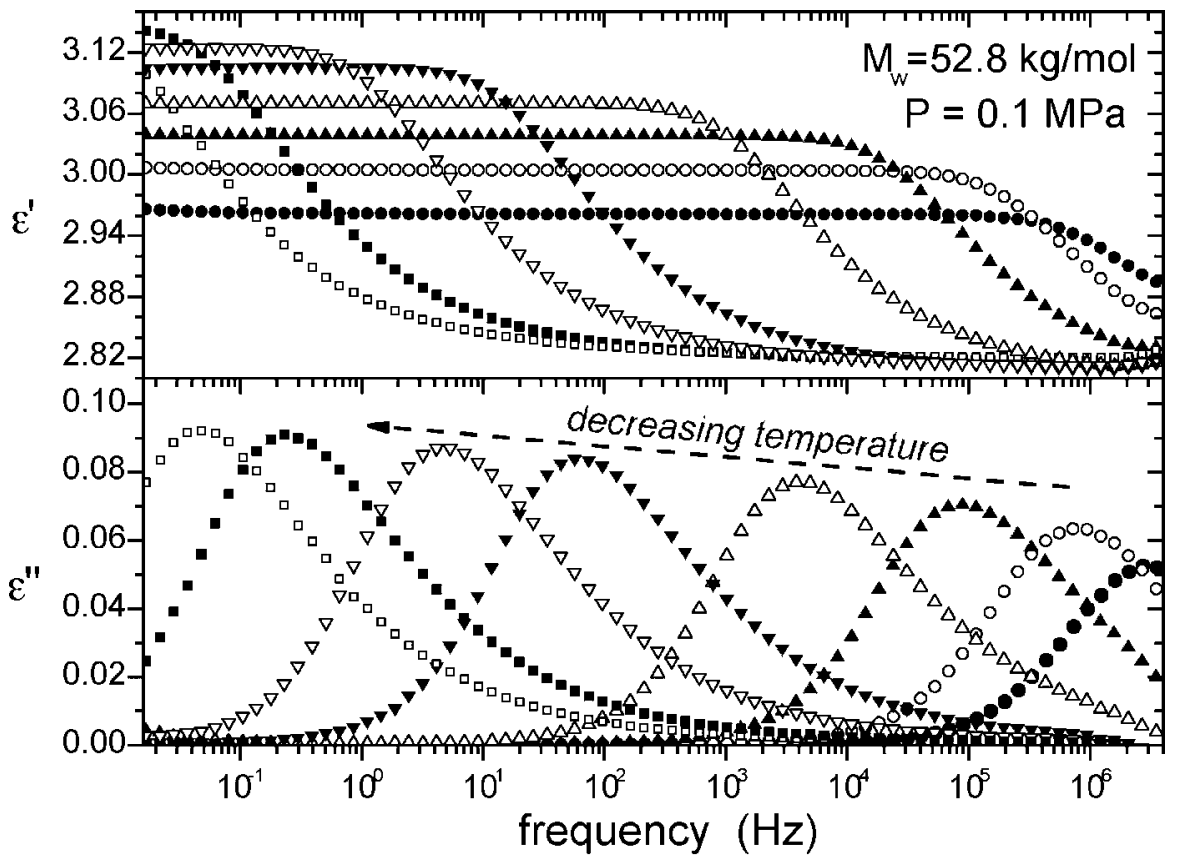

FIG. 1. - Representative dielectric constant and loss curves for PMPS $\left(\mathrm{M}_{\mathrm{w}}=12.6 \mathrm{~kg} / \mathrm{mol}\right)$ at $\mathrm{P}=0.1 \mathrm{MPa}$ and $\mathrm{T}=289(\mathbf{\bullet}) ; 281(\bigcirc) ; 273(\mathbf{\Delta}) ; 265(\triangle) ; 257(\boldsymbol{\nabla}) ; 253(\nabla) ; 249(\mathbf{\square}) ;$ and $247 \mathrm{~K}(\square)$. 


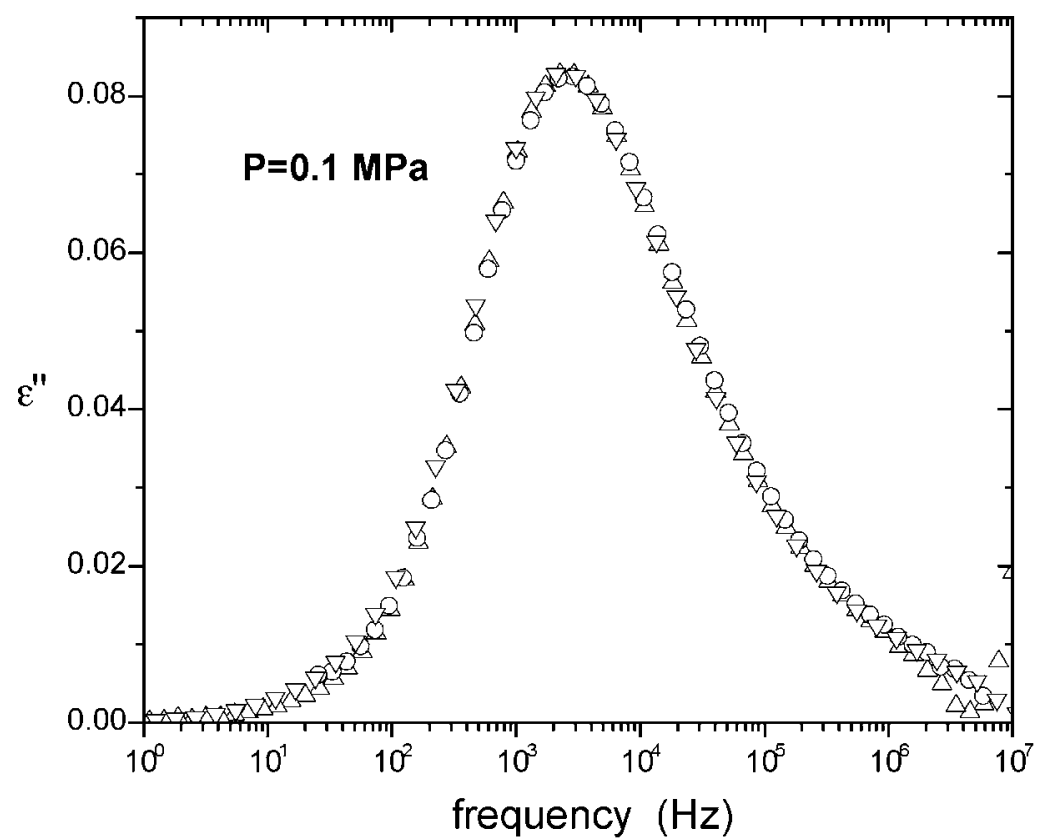

FIG. 2. - Dispersion in the dielectric loss measured at atmospheric pressure for PMPS with $\mathrm{M}_{\mathrm{w}}=12.6(\bigcirc$ at $\mathrm{T}=258 \mathrm{~K}) ; 23.4(\nabla$ at $\mathrm{T}=259 \mathrm{~K}) ;$ and $121 \mathrm{~kg} / \mathrm{mol}(\triangle$ at $\mathrm{T}=264 \mathrm{~K})$.

The curves for the two lower molecular weights were shifted slightly to obtain superimposition.

The change with temperature in the dielectric strength is shown in Figure 3 for the same PMPS as in Figure 1. The decreasing $\Delta \varepsilon$ reflects two effects, an inverse proportionality to temperature and a direct proportionality to the number density of chain units. ${ }^{20}$ The latter, of course, decreases with increasing $\mathrm{T}$.

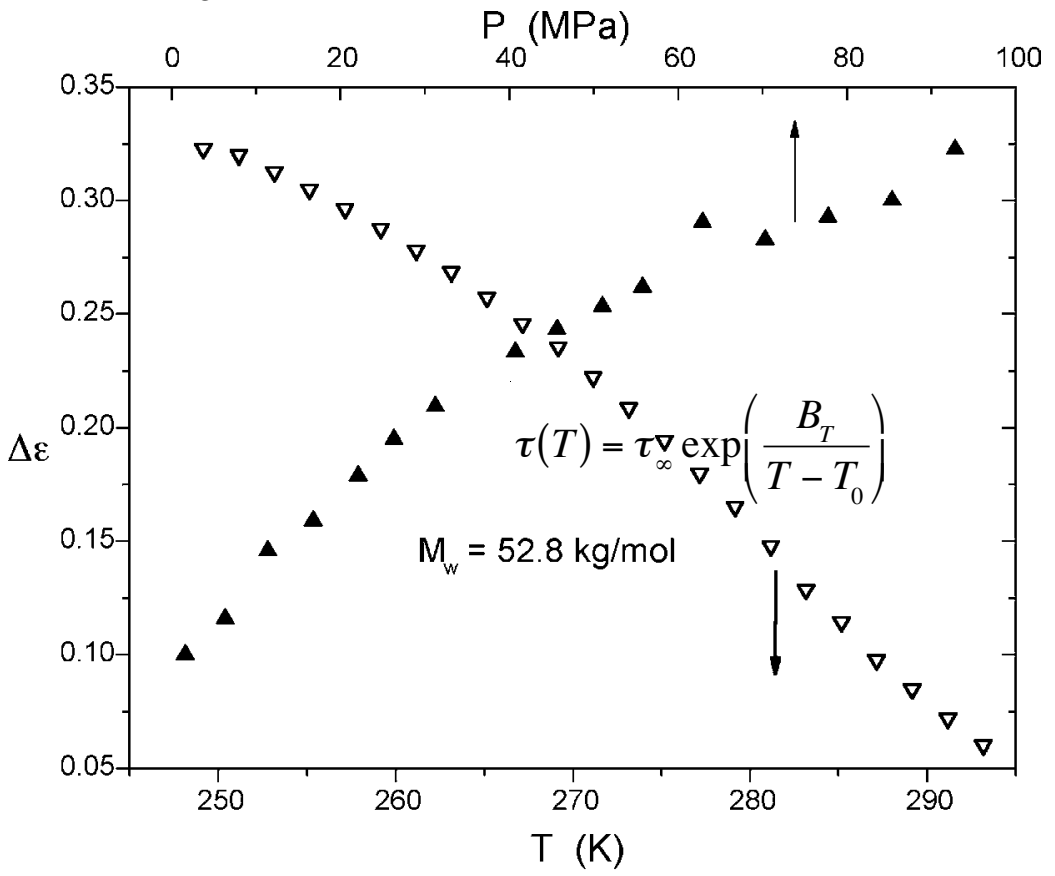

FIG. 3. - Dielectric strength for $\alpha$-relaxation for PMPS $\left(\mathrm{M}_{\mathrm{w}}=52.8 \mathrm{~kg} . \mathrm{mol}\right)$ at atmospheric pressure $(\nabla)$ and at $278 \mathrm{~K}(\boldsymbol{\Delta})$. 
A relaxation time can be defined as $\tau=1 / 2 \pi f_{p}$, where $f_{p}$ is the frequency of the maximum in the dielectric loss. These local segmental relaxation times are plotted in Figure 4 for all samples. It can be seen that beyond $c a .50 \mathrm{~kg} / \mathrm{mol}$ in molecular weight, the relaxation times become independent of $\mathrm{M}_{\mathrm{w}}$. The two PMPS having the shortest chain lengths exhibit substantially smaller $\tau$ at any given temperature. These dielectric relaxation times were fitted to the Vogel-Fulcher equation

$$
\tau(T)=\tau_{\infty} \exp \left(\frac{B_{T}}{T-T_{0}}\right)
$$

which is equivalent to the WLF equation. ${ }^{21}$ Since the $\mathrm{T}_{\mathrm{g}}$ 's of the samples vary, no a priori judgement can be made about the relative dependence on temperature of the relaxation times for the different samples.

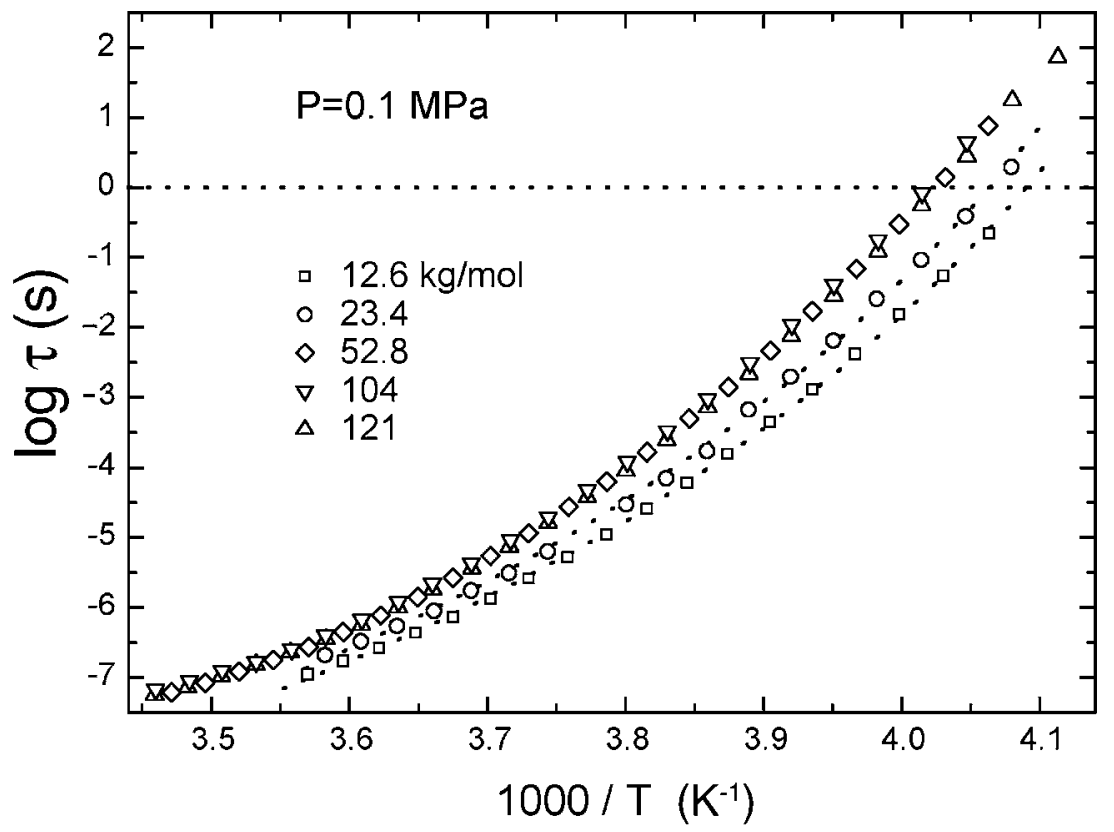

FIG. 4. - Isobaric $(\mathrm{P}=0.1 \mathrm{MPa})$ dielectric $\alpha$-relaxation times for PMPS of the indicated molecular weights. The lines through the data points are the fits to Equation 1.

It is well established that the breadth of the local segmental relaxation function for polymers, as well as small molecule glass formers, correlates with the $\mathrm{T}_{\mathrm{g}}$-normalized temperature dependence of the relaxation times. Thus for PMPS, the fact that the breadth of the loss peak does not depend on molecular weight (Figure 2 ) leads to the expectation that the quantity $\tau\left(\mathrm{T} / \mathrm{T}_{\mathrm{g}}\right.$ ) will be independent of $M_{w}$. We test this by replotting the data from Figure 4, using $T_{g} / T$ as the temperature variable. We take $T_{\mathrm{g}}$ to be the temperature at which $\tau=1 \mathrm{~s}$, using the fits to Equation 1 to interpolate the data. For the lowest molecular weight PMPS, some extrapolation (about one-third of a decade) using the fitted Vogel-Fulcher equation is necessary to reach $\tau=1 \mathrm{~s}$. The obtained values of $T_{g}$ are listed in Table I. As can be seen in Figure 5, this normalization causes the relaxation data to become coincident, consistent with the expectation drawn from Figure 2. 
TABLE I

DieLECTRIC RELAXATION RESULTS FOR POLYMETHYLPHENYLSILOXANES

\begin{tabular}{cccc}
\hline $\begin{array}{c}\mathrm{M}_{\mathrm{w}} \\
(\mathrm{kg} / \mathrm{mol})\end{array}$ & $\begin{array}{c}\mathrm{T}_{\mathrm{g}}{ }^{\mathrm{a}} \\
(\mathrm{K})\end{array}$ & $\mathrm{m}^{\mathrm{a}}$ & $\begin{array}{c}\Delta \mathrm{V}^{\mathrm{b}} \\
(\mathrm{ml} / \mathrm{mol})\end{array}$ \\
\hline 12.6 & 244.5 & 93 & $424 \pm 7$ \\
\hline 23.4 & 246.1 & 92 & $423 \pm 10$ \\
\hline 52.8 & 248.6 & 94 & $437 \pm 7$ \\
\hline 104. & 248.9 & 97 & $436 \pm 4$ \\
\hline 121. & 248.3 & 95 & $436 \pm 7$ \\
\hline
\end{tabular}

${ }^{\mathrm{a}} \tau=1 \mathrm{~s}$ and $\mathrm{P}=0.1 \mathrm{MPa}$.

${ }^{\mathrm{b}} \tau=1 \mathrm{~s}$ and $270<\mathrm{T}(\mathrm{K})<285$.

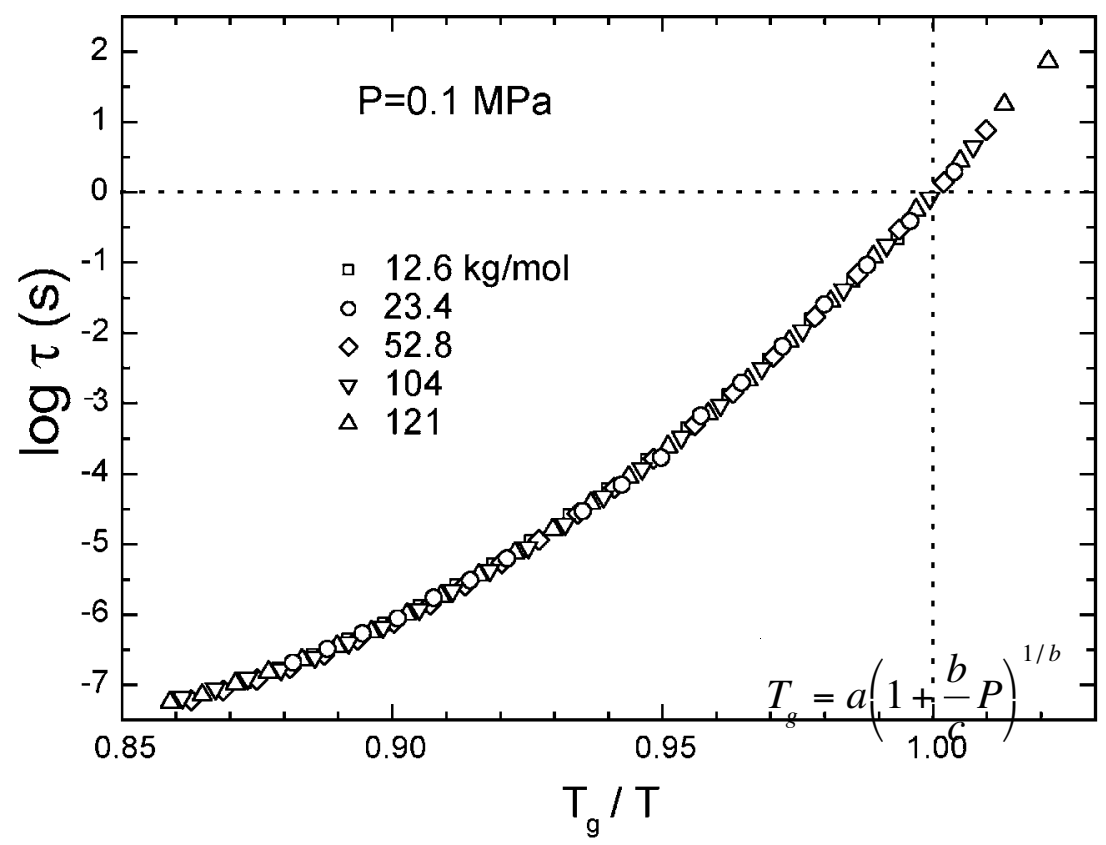

FIG. 5. - Dielectric relaxation times from Figure 4 plotted versus reciprocal temperature normalized by the temperature at which $\tau=1 \mathrm{~s}$

A useful metric to parameterize the temperature dependence is the slope at $\mathrm{T}_{\mathrm{g}}$ of the $\mathrm{T}_{\mathrm{g}}$-normalized Arrhenius curve. This steepness index (or "fragility" ${ }^{\text {) }}$ can be calculated directly from the Vogel-Fulcher fitting parameters as

$$
\left.m \equiv \frac{d \log (\tau)}{d\left(T_{g} / T\right)}\right|_{T=T_{g}}=\log (e) \frac{B_{T} / T_{g}}{\left(1-T_{0} / T_{g}\right)^{2}}
$$

with the result for each sample listed in Table I. The mean value for all molecular weights is $\mathrm{m}=94 \pm 3$. This is intermediate between the available literature values, obtained on only single samples of PMPS. ${ }^{18,22}$ 


\section{EFFECT OF TEMPERATURE AND PRESSURE ON SEGMENTAL RELAXATION 1111}

The measurements on PMPS can be contrasted with results for polydimethylsiloxane (PDMS), which has the same backbones, but with a smaller pendant group. For PDMS, the parameter $\mathrm{m}$ is also independent of the molecular weight. ${ }^{17}$ However, the pendant phenyl ring of PMPS promotes steric interactions and consequently enhanced intermolecular cooperativity. This leads to a broadening of the segmental relaxation function in comparison to PDMS, as well as a more rapid change of $\tau$ with $\mathrm{T}_{\mathrm{g}}$-normalized temperature.

\section{ELEVATED PRESSURE RESULTS}

In Figure 6, representative dielectric spectra measured at various pressures and $278 \mathrm{~K}$ for the lowest molecular weight PMPS are displayed. Qualitatively, the effect of increasing hydrostatic pressure is similar to that of decreasing temperature. There is a systematic shift toward lower frequency, along with an increase in the dielectric strength (Figure 3).

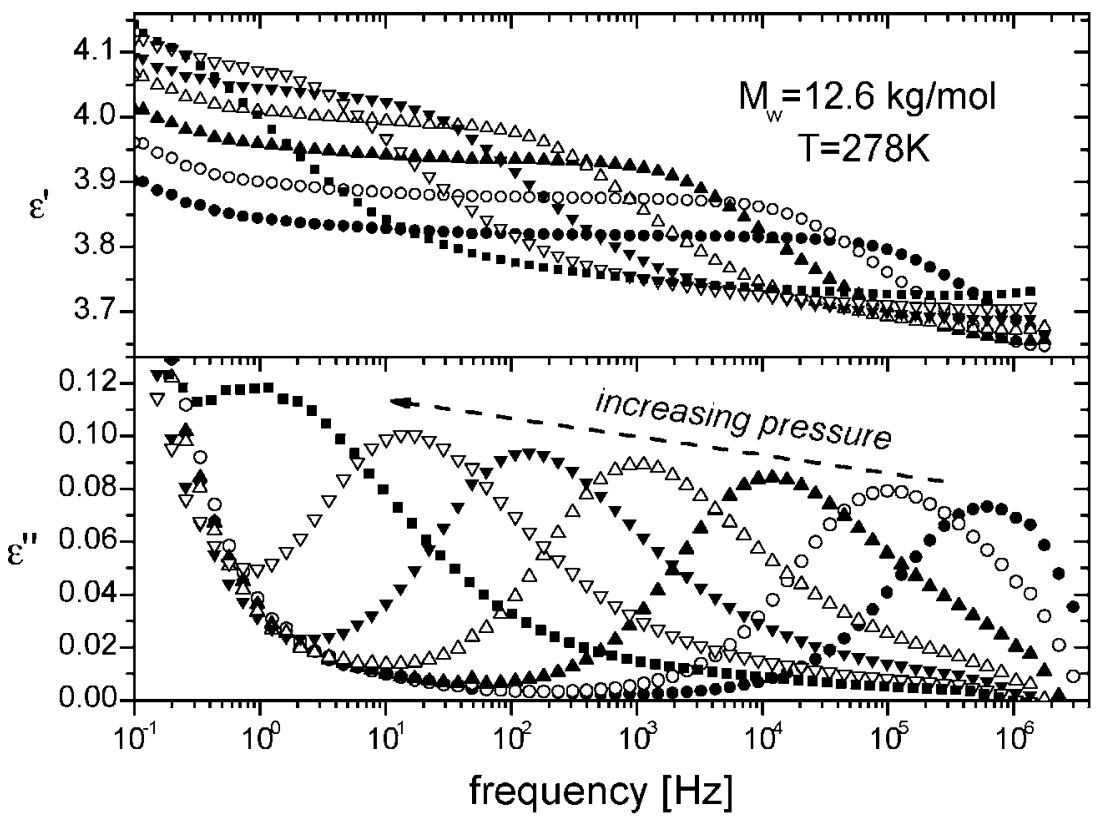

FIG. 6. - Representative dielectric constant and loss curves for PMPS $\left(\mathrm{M}_{\mathrm{w}}=12.6 \mathrm{~kg} / \mathrm{mol}\right)$ at $278 \mathrm{~K}$ and $\mathrm{P}=5.9(\bullet) ; 26.5(\bigcirc) ; 46.5(\mathbf{\Delta}) ; 66.6(\triangle) ; 81.4(\mathbf{\nabla}) ; 96.3(\nabla)$; and $111.5 \mathrm{MPa}(\mathbf{\square})$.

In the same fashion as the isobaric results shown in Figure. 2, the dispersions measured for the different molecular weight samples are equivalent, when compared at pressures for which their respective $\tau$ are equivalent. This is illustrated in Figure 7 for the PMPS having the lowest and highest $\mathrm{M}_{\mathrm{w}}$. This implies that the effect of pressure on the relaxation times will be independent of molecular weight, given the correlation, described above, of the latter with the shape of the relaxation function. ${ }^{1-3}$ 


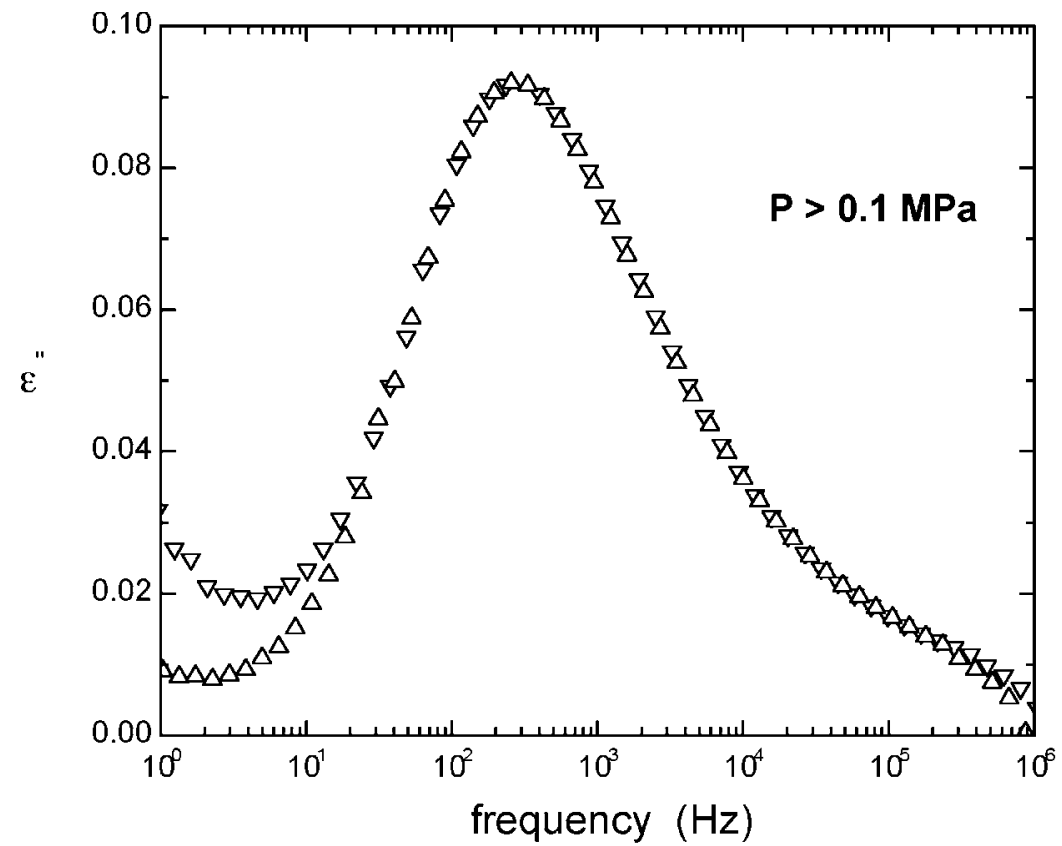

FIG. 7. - Dispersion in the dielectric loss measured for PMPS having $\mathrm{M}_{\mathrm{w}}=12.6 \mathrm{~kg} / \mathrm{mol}$ $(\nabla$, at $\mathrm{T}=278 \mathrm{~K}$ and $\mathrm{P}=7.64 \mathrm{MPa})$ and $\mathrm{M}_{\mathrm{w}}=121 \mathrm{~kg} / \mathrm{mol}(\triangle$, at $\mathrm{T}=274 \mathrm{~K}$ and $\mathrm{P}=45.8 \mathrm{MPa})$.

The latter curve was shifted slightly to obtain superimposition. The low frequency tail seen in the spectrum of the higher molecular weight sample reflects the dc-conductivity contribution to the loss.

Again using the frequency of the maximum in the dielectric loss peaks, we obtain the local segmental relaxation times, with results for three samples displayed as a function of pressure in Figure 8. Results for the other two PMPS are very similar to the data for $\mathrm{M}_{\mathrm{w}}=52.8 \mathrm{~kg} / \mathrm{mol}$. There have been various equations proposed to describe the pressure dependence of $\tau$. $^{23-27}$ Substituting $\mathrm{P}^{-1}$ for $\mathrm{T}$ in Equation 1, a pressure variant of the Vogel-Fulcher equation can be obtained $^{23,28}$

$$
\tau=\tau_{\infty} \exp \left(\frac{B_{P} P}{P_{0}-P}\right)
$$

Using the value of $\tau_{\infty}$ determined from the temperature dependence of $\tau$ at ambient pressure, this function describes the high pressure data well, as shown in Figure 8. 


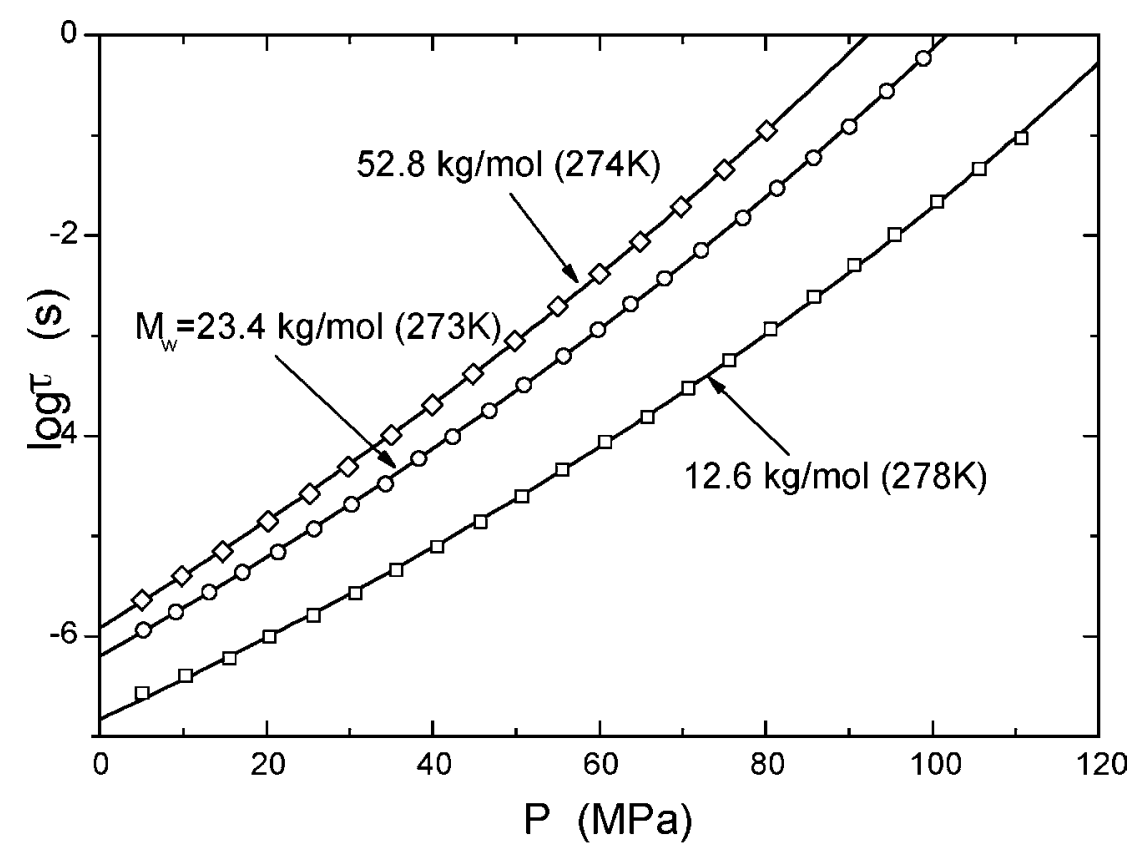

FIG. 8. - Isothermal dielectric $\alpha$-relaxation times for PMPS of the indicated molecular weights at the indicated temperatures. The lines through the data points are the fits to Equation 3.

A useful parameter to characterize the pressure dependence of the relaxation times is the activation volume, defined as $\left.\Delta V^{\#} \equiv \frac{R T}{\log (e)}\left(\frac{d \log \tau}{d P}\right)\right|_{T}$. Having the units of volume, $\Delta V^{\#}$ is interpreted as the volume of the intermediate "transition" state during the relaxation process. ${ }^{8}$ In terms of Equation 3,

$$
\Delta V^{\#} \equiv \frac{R T}{\log (e)} \frac{B_{P} P_{0}}{\left(P_{0}-P\right)^{2}}
$$

We use $\Delta V^{\#}$ herein without implying any conformity to a free volume interpretation of the glass transition. For each molecular weight, we calculate $\Delta V^{\#}$ at a pressure for which $\tau=1 \mathrm{~s}$ at the measurement temperature. Over our limited range of temperatures, the variation of $\Delta V^{\#}$ with temperature is negligible. The obtained results are listed in Table I. Note that the activation volume is almost a factor of 4 larger than the molar volume (the latter calculated from Reference 29). This large magnitude is likely related to the strong effect of volume, relative to thermal energy, on the magnitude of the relaxation times. ${ }^{30}$

Any dependence of the activation volume on $\mathrm{M}_{\mathrm{w}}$ is less than the scatter in its determination; that is, the pressure dependence of the relaxation times of PMPS are independent of molecular weight. Likewise, the pressure coefficient of $\mathrm{T}_{\mathrm{g}}$ is also invariant to molecular weight. By shifting the $\mathrm{T}_{\mathrm{g}}$ of the two lower molecular weight samples upwards by 2.5 and 4.1 degrees, respectively (see Table I), we can superpose the glass transition data. This is shown in Figure 9, wherein the glass transition temperature has a single relationship to pressure. It is interesting that the configurational freedom conferred by chain ends, although causing a decrease in $\mathrm{T}_{\mathrm{g}}$ with decreasing $\mathrm{M}_{\mathrm{w}}$, does not affect the response of the material to pressure changes. 


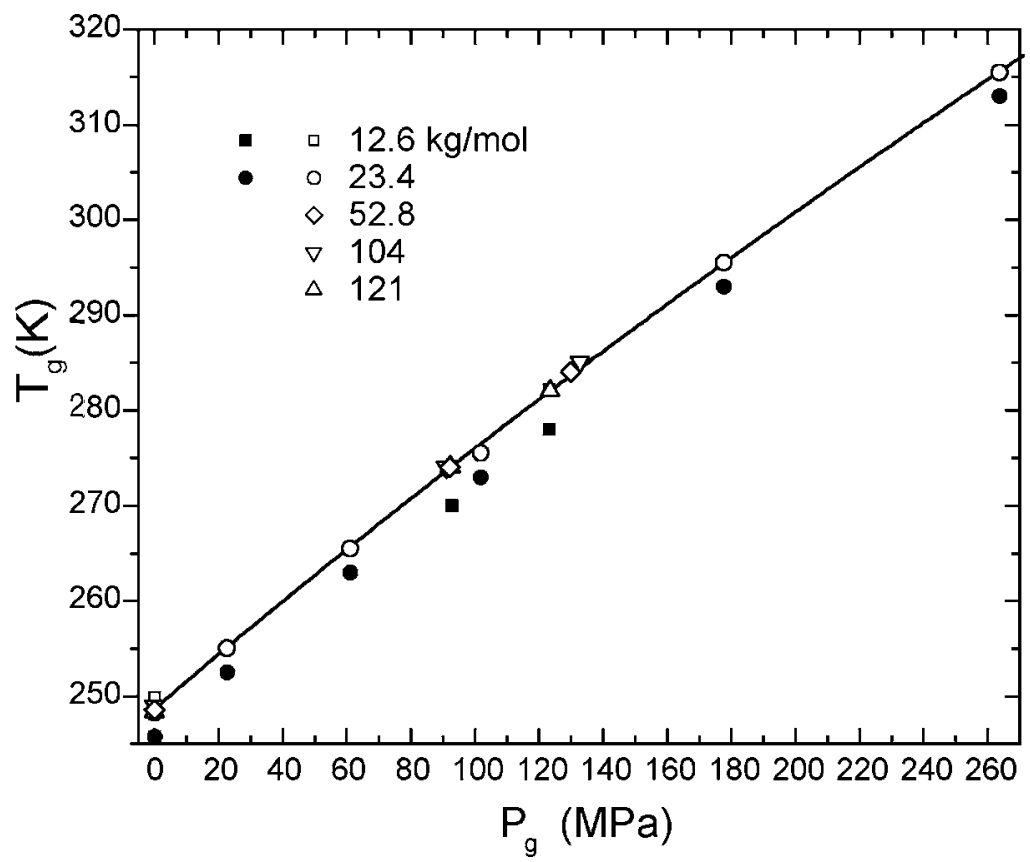

FIG. 9. - Temperature at which the dielectric relaxation time equals $1 \mathrm{~s}$ as a function of pressure for PMPS of the indicated molecular weights. The values for the two lowest molecular weight samples (solid symbols) have been shifted upward by 2.5 and 4.1 degrees, respectively, (hollow symbols) to coincide with the $\mathrm{T}_{\mathrm{g}}$ 's of the higher molecular weight PMPS. The solid line represents the fit to Equation 5.

Commonly, the pressure coefficient of the glass transition temperature is described using the Andersson equation ${ }^{31}$

$$
T_{g}=a\left(1+\frac{b}{c} P\right)^{1 / b}
$$

in which a is essentially the atmospheric pressure value of $T_{g}$, and $b$ and $c$ are material constants. Although originally an empirical fitting function, this equation can be derived from the Avramov model of structural relaxation. ${ }^{32}$ Fitting Equation 5 to the data in Figure 9, we obtain a $=248.7$ $\pm 0.2 \mathrm{~K} ; \mathrm{b}=2.01 \pm 0.15 ;$ and $\mathrm{c}=862 \pm 15 \mathrm{MPa}$. In the limit of zero pressure, $\mathrm{dT}_{\mathrm{g}} / \mathrm{dP}=0.289 \pm$ $0.005 \mathrm{~K} / \mathrm{MPa}$, independent of molecular weight. This dependence on pressure becomes weaker with increasing pressure.

\section{CONCLUSIONS}

The local segmental relaxation properties of polymethylphenylsiloxanes of various molecular weights were investigated using dielectric spectroscopy. The Vogel-Fulcher equation and its pressure analog described well the relaxation times' respective dependences on temperature and pressure. Neither dependence exhibited any variation with chain length. Accordingly, in conformance with the well-established correlation of the relaxation time with the shape of the relaxation function, the latter was found to be invariant to molecular weight. 


\section{EFFECT OF TEMPERATURE AND PRESSURE ON SEGMENTAL RELAXATION 1115}

\section{ACKNOWLEDGEMENTS}

The work at NRL was supported by the Office of Naval Research. J. Ziolo acknowledges financial support by State Committee for Scientific Research (KBN; Poland) under Project No.2PO3B 03323

\section{REFERENCES}

${ }^{1}$ R. Bohmer, K. L. Ngai, C. A. Angell, and D. J. Plazek, J. Chem. Phys. 99, 4201 (1993).

${ }^{2}$ C. M. Roland and K. L. Ngai, Macromolecules 24, 5315 (1991); 25, 1844 (1992).

${ }^{3}$ C. M. Roland and K. L. Ngai, Macromolecules 25, 5765 (1992).

${ }^{4}$ K. L. Ngai and C. M. Roland, Macromolecules 26, 6824 (1993).

${ }^{5}$ C. M. Roland, Macromolecules 27, 4242 (1994).

${ }^{6} \mathrm{C}$. M. Roland, Macromolecules 25, 7031 (1992).

${ }^{7}$ S. Takahara, M. Ishikawa, O. Yamamuro, and T. Matsuo, J. Phys. Chem. B 103, 792 (1999).

${ }^{8}$ G. Floudas, "Broadband Dielectric Spectroscopy," F. Kremer and A. Schönhals, Eds., Springer-Verlag:

Berlin, Heidleberg and New York, 2003, ch. 8.

${ }^{9}$ S. Jiang, W. Jiang, B. A. Wolf, L. An, and B. Jiang, Macromolecules 35, 5727 (2002).

${ }^{10}$ C. M. Roland, R. Casalini, and M. Paluch, Chem. Phys. Lett. 367, 259 (2003).

${ }^{11}$ R. Casalini, M. Paluch, and C.M. Roland, J. Chem. Phys. 118, 5701 (2003).

${ }^{12}$ S. D. Hotston, D. B. Adolf, and K. Karatasos, J. Chem. Phys. 115, 2359 (2001).

${ }^{13}$ G. Floudas, C. Gravalides, T. Reisinger and G. Wegner, J. Chem Phys. 111, 9847 (1999).

${ }^{14}$ M. Paluch, R. Casalini, and C. M. Roland, Phys. Rev. B 66, 092202 (2002).

${ }^{15}$ C. M. Roland and P. G. Santangelo, J. Non-Cryst. Solids 307-310, 835 (2002).

${ }^{16}$ M. J. Schroeder and C. M. Roland, Macromolecules 35, 2676 (2002).

${ }^{17}$ C. M. Roland and K. L. Ngai, Macromolecules 29, 5747 (1996).

${ }^{18}$ M. Paluch, C. M. Roland, and S. Pawlus, J. Chem. Phys. 116, 10932 (2002).

${ }^{19}$ M. Paluch, S. Pawlus, and C. M. Roland, Macromolecules 35, 7338 (2002).

${ }^{20}$ N. J. McCrum, B. E. Read, and G. Williams, "Anelastic and Dielectric Effects in Polymeric Solids," Dover Publications, Inc., Miniola, NY, 1991.

${ }^{21}$ J. D. Ferry, "Viscoelastic Properties of Polymers,” Wiley, New York, $3^{\text {rd }}$ ed. 1980.

${ }^{22}$ D. J. Plazek and K. L. Ngai, Macromolecules 24, 1222 (1991).

${ }^{23}$ G. P. Johari and E. Whalley, Faraday Symp. Chem. Soc. 6, 23 (1972).

${ }^{24}$ G. Fytas, Th. Dorfmuller, and C.H. Wang, J. Phys. Chem. 87, 50411 (1983).

${ }^{25}$ R. Casalini, S. Capaccioli, M. Lucchesi, P. A. Rolla, and S. Corezzi, Phys. Rev. E 63, 031207 (2001).

${ }^{26}$ I. J. Avramov, J. Non-Cryst. Solids 262, 258 (2000).

${ }^{27}$ J. T. Bendler, J. J. Fontanella, and M. F. Shlesinger, Phys. Rev. Lett. 87, 195503 (2001).

${ }^{28}$ M. Paluch, S. J. Rzoska, P. Habdas, and J. Ziolo, J. Phys. Condens. Matter 10, 4131 (1998).

${ }^{29}$ A. C. M. Kuo in "Polymer Data Handbook;” J. E. Mark, Ed.; Oxford: New York, 1999.

${ }^{30}$ M. Paluch, R. Casalini, A. Patkowski, T. Pakula, C. M. Roland, Phys. Rev. E, in press.

${ }^{31}$ S. P. Andersson and O. Andersson, Macromolecules 31, 2999 (1998).

${ }^{32}$ C. M. Roland and R. Casalini, Macromolecules 36, 1361 (2003). 